\title{
Rehabilitation Research at the National Institutes of Health: Moving the Field Forward (Executive Summary)
}

Neurorehabilitation and

Neural Repair

2017, Vol. 3I(4) 304-314

(C) The Author(s) 2017

Reprints and permissions: sagepub.com/journalsPermissions.nav DOI: I0.1I77/I5459683।7698875 journals.sagepub.com/home/nnr

\author{
Walter R. Frontera, MD, PhD ${ }^{1,2}$, Jonathan F. Bean, MD, MS, MPH ${ }^{3,4}$, \\ (SAGE \\ Diane Damiano, $\mathbf{P h D}^{5}$, Linda Ehrlich-Jones, $\mathbf{P h D}^{6}$, Melanie Fried-Oken, $\mathbf{P h D}^{7}$, \\ Alan Jette, $\mathbf{P h D}^{8}$, Ranu Jung, $\mathbf{P h D}^{9}$, Rick L. Lieber, $\mathbf{P h D}^{6}$, James F. Malec, $\mathbf{P h D}^{10}$, \\ Michael J. Mueller, $P^{\prime} D^{11}$, Kenneth J. Ottenbacher, $P^{\prime \prime} D^{12}$, \\ Keith E. Tansey, MD, $\mathrm{PhD}^{13}$, and Aiko Thompson, $\mathrm{PhD}^{14}$
}

Approximately 53 million Americans live with a disability. For decades, the National Institutes of Health (NIH) has been conducting and supporting research to discover new ways to minimize disability and enhance the quality of life of people with disabilities. After the passage of the Americans With Disabilities Act, the NIH established the National Center for Medical Rehabilitation Research with the goal of developing and implementing a rehabilitation research agenda. Currently, a total of 17 institutes and centers at NIH invest more than $\$ 500$ million per year in rehabilitation research. Recently, the director of NIH, Dr Francis Collins, appointed a Blue Ribbon Panel to evaluate the status of rehabilitation research across institutes and centers. As a follow-up to the work of that panel, NIH recently organized a conference under the title "Rehabilitation Research at NIH: Moving the Field Forward." This report is a summary of the discussions and proposals that will help guide rehabilitation research at NIH in the near future.

The conference took place at the NIH Campus on May 25 and 26, 2016. It was cosponsored by The Eunice Kennedy Shriver National Institute of Child Health and Human Development, the National Institute of Biomedical Imaging and Bioengineering, the National Institute of Neurological Diseases and Stroke, the National Institute of Nursing Research, the National Institute on Deafness and other Communication Disorders, the National Center for Complementary and Integrative Health, and the Office of Disease Prevention. The main objectives of the conference were to (1) discuss the current NIH portfolio in rehabilitation research, (2) highlight advances in rehabilitation research supported by NIH, and (3) provide an opportunity for scientists and the general public to comment on gaps in knowledge, opportunities for training, and infrastructure needs. The program included a total of 13 expert panels, 4 remarks by NIH leaders, a consumer keynote, a town hall, a poster session, and the use of social media to disseminate information in real time. The following is a summary of the discussion, and the subheadings correspond to the title of the expert panels.

\section{Rehabilitation Across the Life Span}

(Moderator: Alan Jette, PhD, Boston University; Panelists: Andrea Cheville, MD, Mayo Clinic; Jonathan Bean, MD, Boston University; Shari Wade, PhD, Cincinnati Children's Hospital Medical Center)

The theme of this session was moving rehabilitation interventions from a traditional "one-and-done" isolated model of care to one where rehabilitation interventions are integrated into the mainstream of health care. The speakers addressed integrated care approaches in cancer care, primary care, and pediatric rehabilitation.

Barriers to integrating function-directed care into the comprehensive management of progressive diseases, particularly those with a heavy treatment burden, were identified. Cancer

\footnotetext{
'Department of Physical Medicine and Rehabilitation, Vanderbilt University Medical Center, Nashville, TN, USA

${ }^{2}$ Department of Physical Medicine, Rehabilitation, and Sports Medicine, University of Puerto Rico School of Medicine, San Juan, Puerto Rico

${ }^{3}$ Harvard Medical School, Boston, MA, USA

${ }^{4} \mathrm{New}$ England GRECC, VA Boston Healthcare System, MA, USA

${ }^{5}$ National Institutes of Health, Bethesda, MD, USA

${ }^{6}$ Northwestern Feinberg Medical School and Rehabilitation Institute of Chicago, IL, USA

${ }^{7}$ Oregon Health \& Science University, Portland, OR, USA

${ }^{8}$ Boston University, MA, USA

${ }^{9}$ Florida International University, Miami, FL, USA

${ }^{10}$ Indiana University School of Medicine and Rehabilitation Hospital of Indiana, Indianapolis, IN, USA

"Washington University School of Medicine, St Louis, MO, USA

${ }^{12}$ University of Texas Medical Branch, Galveston, TX, USA

${ }^{13}$ Jackson Veteran's Administration Medical Center, MS, USA

${ }^{14}$ Medical University of South Carolina, Charleston, SC, USA
}

This article is being published almost simultaneously in the following 6 journals: American Journal of Occupational Therapy, American Journal of Physical Medicine and Rehabilitation, Archives of Physical Medicine and Rehabilitation, Neurorehabilitation and Neural Repair, Physical Therapy, and Rehabilitation Psychology.

\section{Corresponding Author:}

Walter R. Frontera, MD, PhD, Vanderbilt University School of Medicine, Vanderbilt University Medical Center, 220I Children's Way, Suite 1318, Nashville, TN 37212, USA

Email:walter.frontera@vanderbilt.edu

Copyright ( $\odot 2017$ The Author(s); published by SAGE Publishing. This is an open-access article distributed under the terms of the Creative Commons Attribution NonCommercial-No Derivatives License 4.0 (CCBY-NC-ND), where it is permissible to download and share the work provided it is properly cited. The work cannot be changed in any way or used commercially without permission from the journal. 
was used an exemplar of the simultaneously dynamic and insidious nature of disablement in chronic illness. Collaborative care approaches, including telecare, validated for pain and depression management, was considered a promising means to proactively and patient-centrically address cancer-related disablement. Current research in cancer rehabilitation suggests that challenges revolve around issues such as patient selection and timing, when and how to intervene, limitations of linear impairment-to-disability models (with multiple mild impairments the norm), and competition with disease-modifying therapies. Although functional limitations are prevalent (seen in $65 \%$ of all cancer patients), rehabilitation interventions remain underused. In contrast to ischemic and traumatic injuries, rehabilitation interventions in patients with cancer are less prescriptive, more negotiable, and subject to patient preferences. Current care delivery overwhelmingly emphasizes primary disease management.

Another presentation focused on limitations with mobility tasks, such as walking, rising from a chair, or climbing stairs, as a signal condition identifying older adult primary care patients at an increased risk for disability, morbidity, and death. It was discussed how rehabilitative care can play a critical role with older adult primary care patients by developing integrated care paradigms between primary and rehabilitative care providers focused on prevention of mobility decline among older adults. Prevention of adverse health outcomes represents a new conceptual role for rehabilitative care. Research priorities include determining the optimal content and design of preventive rehabilitative care; the potential benefits for patients, families, and health care organizations; and the cost/benefit of such approaches to care.

Traumatic brain injury (TBI) was used as a case example to discuss the need for further research on ways to integrate pediatric rehabilitation into the broader framework of child development. TBI is currently viewed as a discrete event with time-limited consequences, whereas evidence from the TBI Model Systems suggests lifelong physical and cognitive consequences. Long-term pediatric studies are lacking, but existing evidence suggests long-term effects on educational attainment and vocational and social success. However, after the post-acute recovery phase, children with TBI receive little ongoing rehabilitation. TBI-related problems that emerge with shifting developmental demands may go unrecognized or be inaccurately characterized. Families and schools constitute powerful contexts for ongoing rehabilitation and later habilitation. How families function and interact with the child exerts a powerful influence on the recovery trajectory. Interventions need to be developmentally tailored and address the current developmental and neural context. Challenges remain in framing rehabilitation/habilitation as an ongoing process with tuneups at various developmental stages rather than a one-and-done model. A better understanding of adult outcome metrics (eg, education and employment) and long-term burden (disability and life quality) is needed. To reduce heterogeneity and improve prediction, research is needed to better categorize the initial injury/insult along with better understanding of effects on neurodevelopment and how this relates to long-term functional outcomes. Multicenter consortiums are urgently needed to support larger-scale outcome studies and provide an infrastructure to link school and medical data as well as study interventions and management practices more efficiently.

\section{Technology in Rehabilitation: From Cutaneous to Implanted}

(Moderator: Ranu Jung, $\mathrm{PhD}$, Florida International University; Panelists: Leigh Hochberg, MD, PhD, Harvard University; Reggie Edgerton, $\mathrm{PhD}$, University of California, Los Angeles; Joseph Rizzo, MD, Harvard University; Mario Svirsky, PhD, New York University)

Innovation and advances in engineering and computing are having a ubiquitous impact on health and well-being. The purpose of this panel was to discuss the challenges and opportunities for developing technologies that interface with the nervous system at an appropriate level, are user centric and responsive to the ability of the user and their life span, and could provide new neuroscience insights to inform rehabilitation science. The panel also discussed the importance of having appropriate assessment methodologies and comprehensive engagement with regulatory, industry, and clinical partners. The moderator and panelists brought to the discussion their experience as neuroscientists, biomedical engineers, and clinical practitioner, some with personal experience of moving neurotechnology from the laboratory to human studies. Using examples from engineering of cochlear and visual prosthetic devices and brain, spinal cord, and peripheral nerve interfaces, they discussed the role of technology in scientific discovery and recovery and restoration of missing or lost function.

The overall span of the technology that can influence rehabilitation is broad: from assistive devices, rehabilitation robotics, and implanted neuroprostheses to augmented connectivity between people and devices, use of virtual reality environments for training, and use of mobile health and telehealth platforms for deployment of rehabilitative therapies. The panel discussions focused on implanted neuroprostheses. Advances in neurotechnology will allow us to better access information about the living system at multiple scales, from cellular to behavioral. Improved understanding of the endogenous activity patterns of neural activity could help guide the design of neuroprostheses that can more precisely influence and modify the neural activity to initiate and sustain long-term beneficial neuroplasticity leading to repair or recovery. Design, development, and deployment of the neuroprostheses that form biohybrid systems with the living body has many challenges.

A major challenge in the deployment of neuroprostheses that effect recovery is to make the neuroprostheses adaptive and patient centric. The panel discussed that the scheduling (timing) for introducing rehabilitation technology after a traumatic event to patients is very important. In addition, 
whether all the capabilities for the neurotechnology should be introduced immediately or in a controlled sequential manner after deployment has to be considered. For example, after a bilateral sequential implantation of cochlear implants, should they be deployed sequentially or together? To restore function after incomplete spinal cord injury (SCI), should epidural stimulation be conducted in parallel with or before treadmill training? Recovery of function is very patient specific and may confound assessment of the effectiveness of different neural stimulation paradigm interventions. To design appropriate rehabilitation therapies, conduction of scientific studies in tandem with technology development would be highly beneficial. This in itself raises new challenges.

Several of the technological interventions could require extensive development, and the underlying science of rehabilitation may be insufficient to support the use of these technologies for larger-scale human use. It is essential that early development of neurotechnologies, including the scientific studies that provide the evidence, are conducted with close consultation of the regulatory bodies such as the Food and Drug Administration. The safety and reliability of small early-feasibility trials need to be considered. In this context, the panel suggested that for sequential improvements in technology, a modular design be used. In addition, giving the participant at least some control over use of the technology, as needed, was considered important. This requires the development of a regulatory acceptance pathway. There was considerable discussion on the design of study protocols with small numbers of enrolled participants. Each participant's own abilities with turning on or shutting off the device could be used as an internal control for device evaluation, thereby formalizing and extending the value of small studies. The lack of commercial support for conducting studies with a small number of participants together with the associated legal and regulatory requirements indicates that governmental funding support for technology development and early-feasibility trials is paramount for translation of the neurotechnologies from the laboratory to the clinic.

A key outcome from the panel discussion was that implanted neurotechnologies offer a "precision medicine" approach to rehabilitation. They target specific neural populations. The stimulation paradigms could be combined with other treatments, especially cell therapies, to maximize function. This ability for precision deployment could be further tailored to take advantage of the genetic makeup of the recipient to make it a personalized, adaptive approach to rehabilitation.

\section{Mechanisms and Markers of Activity and Function}

\section{Exercise, Plasticity, and Mechanism: "How is Rehabilitation Happening?"}

(Moderator: Keith Tansey, MD, PhD, Methodist Rehabilitation Center; Panelists: Rick Lieber, PhD,
Rehabilitation Institute of Chicago; Stephen Seliger, MD, University of Maryland; James Blumenthal, PhD, Duke University)

Rehabilitation interventions are applied to various patient populations with diverse physiological profiles over extended periods with relatively little evidence regarding which interventions are doing what in whom. Patients with neurological problems need to be characterized better, so that we can identify and analyze responders versus nonresponders. Monitoring tools to ensure that rehabilitation interventions are proceeding toward more normal physiology over time are also needed. Neurological plasticity after injury can be both adaptive and maladaptive, and we need to work to gain the former while limiting the latter. Similarly, skeletal muscle plasticity is important in injury and rehabilitation, but classic measures rarely capture the functionally relevant properties of skeletal muscle. Most plasticity studies focus on muscle active properties such as force generation and fatigue and less so on problems involving passive mechanical properties such as contracture or fibrosis. New areas of investigation in the field include extracellular matrix structure and function and the development of new imaging methods that would permit mesoscale quantitative measures of muscle performance that are objective and clinically relevant. Older adults with chronic kidney disease have impaired neurocognitive function, physical performance, and aerobic capacity. Research has been done on the mechanisms associating kidney disease to physical and cognitive impairment. Exercise training improves neurocognitive function and protects against cognitive decline in chronic renal disease patients. Finally, patients undergoing cardiac rehabilitation benefit from stress management. The Enhancing Cardiac Rehabilitation With Stress Management Training trial shows the beneficial effects of combining stress management training with standard exercise-based cardiac rehabilitation in terms of stress levels, coronary heart disease biomarkers, and clinical outcomes. These findings should be disseminated, and a cardiac rehabilitation program, including stress management, should be made more accessible to patients with coronary heart disease.

The major issues identified and questions raised in this session for further consideration going forward were as follows. We have to address diverse populations (physiologically) in rehabilitation, even within a given diagnosis. We also need to address our lack of mechanistic understanding of interventions in preclinical and clinical scenarios, which makes predicting responders versus nonresponders difficult and makes translation from animal models to humans problematic as well. The idea of tracking progression during an intervention was introduced: Are we generating more normal biology/function or developing "work-arounds" in rehabilitation? The question was raised as to whether we are measuring the right biological markers in our systems, the ones that are actually critical to the pathophysiology/impaired function, so as to develop appropriate interventions. We may need to 
develop better assessment tools (imaging for instance) to understand these issues. We may also need to connect previously unconnected areas of medicine (chronic disease states and their neurological impact, for instance) to make a wider impact with our interventions. Finally, we should partner psychological interventions with rehabilitation interventions to have a greater impact, overall, on human health.

\section{Access to the Lived Environment}

(Moderator: Melanie Fried-Oken, PhD, Oregon Health \& Science University; Panelists: Cole Galloway, $\mathrm{PhD}$, University of Delaware; Maureen Schmitter-Edgecombe, $\mathrm{PhD}$, Washington State University; James Coughlan, PhD, Smith-Kettlewell Eye Research Institute)

This panel presented and discussed evidence that assistive technologies (ATs) provide functional tools to ensure that individuals experience their greatest level of functional independence in daily life. Based on the International Classification of Functioning, Disability, and Health of the World Health Organization, AT is a facilitator for activities and participation for individuals who experience disability and chronic health conditions. The technologies being developed, discussed, and tested by this panel are often mainstream technologies available to the general public that are adapted to meet functional needs and access to daily environments. Devices such as off-the-shelf toy racecars that can provide mobility to children with physical impairments, environmental controls with infrared sensors to support or assess elders with dementia who are aging in place, and application software for touch tablets and mobile phones that guide travelers with visual impairments at traffic intersections were discussed and demonstrated through multimedia presentations.

The panel discussed 3 common themes and a number of challenges to the design, testing, and implementation of ATs, including the following:

1. Participatory action research as a critical element of rehabilitation research. Individuals with disabilities must be included in all stages of hypothesis testing and analysis to ensure content validity. Participatory action research is sensitive to group as well as individual differences (ie, cultural, ethnic, lifestyle diversity) and leads to people having increased control over their lives.

2. The utility of AT for value added to end users and professionals must become a priority for rehabilitation science. Utility measures such as task performance (eg, efficiency and effectiveness of task completion), user satisfaction, and quality of life must become standard. It is challenging to measure value because the user population is extremely heterogeneous in terms of needs, abilities, and preferences. Researchers must determine if it is better to assess utility for a narrow population that is most likely to benefit from AT or a broad population, where only a subset of individuals is likely to benefit. The variability of user population and task conditions can make it very hard and/or costly to get good statistics on utility. Although statistical success is easier to obtain under controlled laboratory conditions, the laboratory conditions do not translate to real-world conditions. Measurement of user satisfaction (or dissatisfaction) and quality of life, constructs that are often used for outcomes, has challenges as well.

3. AT must be scaled, in terms of sustainability and accessibility, to the population. As technology is rapidly advancing, we must try to get at the back end of it even as it gets more complex. For example, as infrared sensors became wireless, laboratories and smart homes needed to adjust, so that our tools are sustainable. For the biggest impact, one goal in technology research and development must include keeping products and services affordable, so they can be accessed by the population who needs them. Likewise, we must increase awareness and benefits of ATs for the general public. The AT must meet the environmental and personal demands of the end users while protecting privacy and maintaining confidentiality and security of personal information.

\section{Individuals, Families, and Community}

(Moderator: Linda Ehrlich-Jones, PhD, Rehabilitation Institute of Chicago; Panelists: Christopher Murtaugh, $\mathrm{PhD}$, Visiting Nurse Service of New York; George Alexopoulos, MD, Cornell University; Sara Czaja, PhD, University of Miami Miller School of Medicine)

Rehabilitation interventions incorporating the home, the family, and the community promote active engagement of patients, family, and community members to achieve increased quality of life for people with disabilities. Psychosocial interventions aimed at reducing poststroke depression and stress rely on 5 integrated components: (1) offer patients action-oriented "new perspective" about recovery; (2) provide an "adherence enhancement structure"; (3) offer a "problem solving structure" to the patient focusing on problems, valued by the patient, and pertinent to daily function; (4) help the patient's family "reengineer its goals, involvement, and plans" to accommodate the patient's disability; and (5) "coordinate care with specialized therapists" with the goal to increase patient participation in rehabilitation and social activities.

The outcomes of patients receiving physical therapy at home for activity-limiting pain, total hip or knee replacements, and implantable cardiac devices show some 
improvement over time. Family caregivers play a critical role in supporting older adults and family members with a chronic disease or disability. Intervention strategies that are aimed at supporting family caregivers and reducing caregiver burden with an emphasis on technology-based interventions are needed to facilitate improved outcomes in people with disabilities. The end goal of incorporating the home, the family, and the community is greater independence and providing opportunities for people with disabilities to actively contribute to their community. Strategies that help individuals to self-manage their disability can lead to achievement or maintenance of positive outcomes. The challenges experienced by caregivers of individuals with disabilities need further attention.

Gaps and opportunities for future research include examination of the impact of sociodemographic influences, including geography, socioeconomic status, education, and language/culture, on rehabilitation success. In addition, development of self-management strategies that can be implemented in community settings to help individuals better understand and manage their disability and achieve or maintain positive quality of life and independence are necessary areas of future research.

\section{Understanding the Context: Environmental Impacts in Rehabilitation}

(Moderator: Michael Mueller, PhD, Washington University School of Medicine; Panelists: James Burke, MD, University of Michigan; Amanda Botticello, PhD, MPH, Kessler Foundation; Patrick Kitzman, PhD, University of Kentucky)

The purpose of this session was to consider how environmental factors affect outcomes in rehabilitation. The "environment" is an important, modifiable, and understudied element in the International Classification of Functioning, Disability and Health framework. An example was provided for patients with diabetes and peripheral neuropathy that illustrated how a conceptual framework had been used to help direct interventions at the environmental level (casting, footwear, community screening, and education) to reduce the rate of lower-extremity amputation. Other, more complex models are being developed to illustrate ways in which race and socioeconomic factors may interact with contextual factors such as caregiver support, transportation, neighborhood environment, and social network to limit access to rehabilitation. Some drivers of racial differences in poststroke disability are modifiable, and we should consider stroke survivor and family level strategies to reduce disability and decrease disparities.

There are links between community context and longterm outcomes for persons with SCI. Community characteristics such as socioeconomic disadvantage, resource deprivation, segregation, and physical inaccessibility likely threaten the physical, psychological, and social functioning gains achieved during rehabilitation. Neighborhood socioeconomic factors affect health and well-being over and above personal characteristics. For example, employment rates for SCI are poor, with rural $<$ suburban $<$ urban. The best prospects for employment and community participation are for those people with SCI and high socioeconomic status in urban environments. The challenge of providing rehabilitation services to people with SCI in rural settings was highlighted with a description of a specific program targeting rural Kentucky, a state at the bottom of several US health outcome measures. The Kentucky Appalachian Rural Rehabilitation Network is working to overcome these barriers and encourages a bidirectional flow of information, providing clear benefits for the community, being accountable and providing long-term commitment (ie, sustainability) to the community.

All presentations and discussion highlighted the fact that interactions between environment and outcomes are highly complex and vary according to location, socioeconomic level, race, age, and disability. Understanding these complex relationships will require further refinement of conceptual models and a variety of research approaches to understand outcomes and devise policy to enhance outcomes. The use of "big data sets" is useful, and the net of these data sets need to be spread even further to capture common concerns across wide geographical areas. Consistent with other sessions, there is a need for common outcome measures, but also for qualitative studies to better understand these themes at an individual level. Finally, another important theme was the need for ongoing support for people with chronic disabilities. Longitudinal research is needed to determine how disability affects people in their environment over time. Intervention should not be "one and done" but should dynamically meet the ongoing and changing needs of people with chronic health problems.

\section{Effective Pathways to Evidence for Rehabilitation}

(Moderator: James Malec, PhD, Indiana University School of Medicine/Rehabilitation Hospital of Indiana; Panelists: Lynn Snyder-Mackler, PT, ScD, University of Delaware; Catherine Lang, PhD, Washington University; Susan Horn, $\mathrm{PhD}$, University of Utah)

This symposium examined phases, options, and challenges in advancing a line of rehabilitation research. Methodologies for addressing challenges were explored as well as for incorporating mechanisms, defining dose, and examining the effectiveness of standard rehabilitation procedures.

Traditional phases in a line of research include idea generation, natural history and/or animal models, early human 
testing for safety and feasibility, efficacy trials, and effectiveness trials. This sequence may be most informative if viewed as iterative and recursive rather than linear. Designs such as the randomized controlled trial (RCT) offer strong internal validity. However, some aspects of the RCT, for example, participant and researcher blinding and development of a viable control condition, may be difficult to implement in rehabilitation research. Other designs, such as large-scale observational or practice-based evidence trials, may offer stronger external validity. Balancing internal and external validity is critical to encourage timely translation into practice. Other considerations and challenges in advancing rehabilitation research include heterogeneity of participants and interventions (which are typically individualized in practice), fidelity assurance, dosing, consideration of nonspecific factors as moderators as opposed to confounders of treatment effect, and the precision of measurement tools used to assess outcomes that are not directly observable and must be assessed by observer or participant rating.

Investigating the underlying mechanisms of action in high-quality clinical trials and observational quasi-experimental studies within rehabilitation research is achievable but fraught with obstacles that do not occur in typical clinical drug trials. Unlike the delivery of an active medication or placebo, rehabilitation interventions are typically multimodal and involve active participation of both the patient and the clinicians. Thus, ensuring fidelity, that is, defining the intervention(s), ensuring that the intervention(s) are reliably applied, and defining the active component(s), is particularly challenging in studies of rehabilitation. Use of fidelity metrics, ideally completed by more than one observer, addresses this challenge. In rehabilitation research, outcomes are often complex, occur across the domains of the International Classification of Function, and include patient-reported as well as performance-based and instrumented outcomes. Consequently, strategic selection and pretrial testing of precision outcome metrics and control conditions are critical.

It is also critical to consider dosing in rehabilitation trials to not waste resources and to eventually improve outcomes. In rehabilitation, dose is an interaction of multiple parameters. Explicit studies of dose-response are necessary to determine essential information about active ingredients, their biological targets and mechanisms of action, and their halflives. As with other elements of high-quality clinical trials, key dosing parameters are best determined through pretrial feasibility study. Methods to determine appropriate dose include (1) careful quantification of the active ingredient, (2) multiple assessments over the course of the intervention, (3) multiple groups receiving different doses, and (4) sophisticated statistical modeling of data across time (eg, hierarchical linear modeling, individual growth curve analysis).

Electronic medical records are collecting detailed patient, treatment, and outcome data now and will do so even more in the future. This information can be used to determine those interventions that are associated with better outcomes for patients with specified sets of characteristics through practice-based evidence study designs. Practicebased evidence is an example of an innovative research methodology that addresses many of the challenges to the traditional RCT posed by rehabilitation research.

This symposium identified a number of challenges to interventional rehabilitation research, including heterogeneity of participants, individualized and complex treatments, balancing internal and external validity, implementing viable control conditions, difficulty blinding participants and researchers, nonspecific treatment moderators, fidelity assurance, and dosing. A greater emphasis on pretrial studies and alternative designs to the traditional RCT offer opportunities to address many of these challenges.

\section{Central and Peripheral Mechanisms of Rehabilitation}

(Moderator: Rick Lieber, PhD, Rehabilitation Institute of Chicago; Panelists: D. Michele Basso, PhD, Ohio State University; Monica Perez, PhD, University of Miami; Mike Boninger, MD, University of Pittsburgh)

In this session, the mechanisms of plasticity in rehabilitation were discussed. The presenters focused on approaches to measuring brain, spinal cord, and skeletal muscle function and discussed how rehabilitation and regenerative therapies could be applied to improve central and peripheral function.

Human skeletal muscle adapts to contractures that occur secondary to stroke and cerebral palsy $(\mathrm{CP})$. Intraoperative structural studies of upper-extremity muscles show that sarcomere length increased, whereas serial sarcomere number decreased dramatically. The extracellular matrix in contractures was deranged (hypertrophic and altered composition) and apparently does not support a functional stem cell niche. Using both flow-assisted cell sorting and immunohistochemistry, it has been demonstrated that satellite cell number (muscle stem cells) is decreased by about $70 \%$ in contractures. This may cause muscle shortening, deranged extracellular matrix, and increased muscle stiffness. Finally, studies of gene expression from these muscles revealed altered transcriptional pathways relative to other models of decreased use such as immobilization, SCI, or spaceflight. Thus, muscle contracture represents a dramatic and unique model that must be understood mechanistically to develop novel treatment approaches.

Studies at the cellular level may explain why SCI rehabilitation can be quite effective in some individuals, whereas others show limited improvement. In rodent models of contusion, the timing to deliver task-specific training and cellular factors that are conducive to motor learning has been determined. These findings suggest that inflammation in cord regions remote to the injury is a barrier to effective 
rehabilitation. In fact, animal models show that training delivered early after SCI during high inflammation worsens function but reducing this inflammation allows robust locomotor recovery using a brief training paradigm. The source and genetic profiles of cellular inflammation have been identified, which may allow development of biomarkers for rehabilitation.

New neuroplasticity protocols are being used in humans with SCI, and noninvasive electrophysiology can be used to guide therapeutic interventions. The corticospinal tract is an important target for motor recovery after SCI. Noninvasive techniques have been used to develop tailored protocols for precise timing of the arrival of descending and peripheral volleys at corticospinal synapses of upper- and lower-limb muscles in humans with chronic partial paralysis. Voluntary motor output depends on the efficacy of synapses between corticospinal axons and spinal motor neurons, which can be modulated by precise timing of central and peripheral neuronal spikes. Thus, noninvasive techniques can be used to develop tailored protocols for precise timing of the arrival of descending and peripheral volleys at corticospinal-spinal motor neuron synapses involved in intrinsic finger muscle function in humans with chronic incomplete SCI. Using electrophysiological measurements by stimulating different levels of the corticospinal pathway in individual subjects, accurate estimates of the time of arrival of action potentials to the muscle have been measured; indeed, latencies of electromyographic responses relied on the generation of action potentials in motor neurons. The results indicate that arrival of presynaptic volleys before motor neuron discharge enhances corticospinal transmission and hand voluntary motor output. In contrast, the reverse order of volley arrival and sham stimulation does not decrease voluntary motor output and electrophysiological outcomes. Overall, these findings demonstrate that spike timing-dependent plasticity of residual corticospinal-spinal motor neuron synapses provides a mechanism to improve motor function after SCI. Modulation of residual corticospinal-spinal motor neuron synapses may present a novel therapeutic target for enhancing voluntary motor output in motor disorders affecting the corticospinal tract.

The integration of principles and approaches in rehabilitation science and regenerative medicine may help us develop innovative and effective methods that promote the restoration of function through tissue regeneration and repair. The application of rehabilitation protocols in combination with cellular therapeutics for the treatment of injured or diseased tissue enhances transplantation efficacy and improves functional outcomes. Although it is clear that the convergence of rehabilitation approaches with regenerative medicine strategies will accelerate the science underlying tissue restoration after injury and disease, collaborative research efforts across the fields of regenerative medicine and rehabilitation are greatly lacking. An NIH reporter search of active funding using the Boolean term regenerative medicine yielded more than 2231 studies. When we modified this search to only include physical medicine and rehabilitation and other health professions, which include physical therapy, occupational therapy, and speech language pathology departments, only a total of 16 grants were displayed. This is remarkable considering that the promotion of tissue healing and regeneration is a primary goal of many rehabilitation interventions. There is, therefore, a great need to expand scientific knowledge, expertise, and methodologies across the domains of rehabilitation science and regenerative medicine, with the ultimate goal of improving the lives of individuals with disabilities.

Gaps in the understanding of mechanisms underlying rehabilitation include the following questions: (1) What has the greatest impact on skeletal muscle strength, the nervous system or the biomechanical manipulation of muscle? (2) Which stem cell populations can rehabilitation professionals realistically manipulate? (3) How can exercise influence the stem cell population? (4) How do bio-scaffolds interact with stem cells? (5) Because the timing of SCI treatment is an important factor in good outcomes, how will we be able to translate animal studies into human treatments? (6) What are the most appropriate strategies for applying regenerative medicine to rehabilitation? (7) Does the cellular state of the central nervous system dictate the response to rehabilitation treatment, or can the right type of exercise modify the cellular environment to create permissive learning conditions?

\section{Bending the Arc of Technology Toward Rehabilitation and Health}

(Moderator: Aiko Thompson, PhD, Medical University of South Carolina; Panelists: Steve Cramer, MD, University of California, Irvine; James Rimmer, $\mathrm{PhD}$, Lakeshore Foundation; Susan Magasi, PhD, University of Illinois at Chicago)

The purpose of this session was to discuss how the integration of technology into rehabilitation, health care, and wellness services can promote better communication between health care professionals and patients and thereby achieve healthy lifestyles and better quality of life.

The use of information and communication technologies eliminates distance barriers and can make rehabilitation and health care services available to people who have limited access to transportation and other access issues. In recent years, digital health (eg, telehealth, telerehabilitation [telerehab], eHealth [health care services delivered or enhanced through the Internet], and mHealth [delivery of health care services via mobile communication devices]) has become a significant part of the health care and health care economy. Digital health funding has been steadily increasing. Tools for developing and implementing mobile 
health care services and research applications are becoming more and more available. It is clear that the use of information and communication technology can broaden rehabilitation and health care research opportunities for researchers and service opportunities for patients. In this session, the speakers provided 3 levels of remote rehabilitation training management: full management (by health care professions), middle-level management, and self-management (by patients). These different levels are not mutually exclusive but are harmonious approaches that allow the patient to transition from one level of management to another, based on his/her progress in recovery and changes in needs for care and services.

Many patients do not receive enough dose of rehabilitation therapy after stroke. Telerehab is ideally suited to maximize the gains from therapy; for instance, telerehab can increase the duration and intensity of therapy and, therefore, contribute to greater functional gains. Pilot studies and clinical trials are ongoing (Cramer) on a home-based telerehab system for patients with stroke. Telerehab also offers the option for a holistic approach to patient care-for example, incorporating education, sensor data collection, and regular structured interactions with therapists.

Other technologies such as eHealth and mHealth can contribute to health promotion emphasizing self-care rather than expert care. In furthering the view that digital health technologies can help overcome existing health care problems (eg, lack of integration and coordination across different disciplines and accessibility barriers), it was suggested that telerehab may prevent well-known postrehabilitation health decline as the patient transitions from dependence to independence. Preliminary findings of the ongoing project (Rimmer), "TExT-ME: Telehealth Exercise Training for Monitoring and Evaluation of Home-Based Exercise," show that home-based tele-exercise interventions can achieve better participant adherence than conventional onsite exercise programs, leading to better health benefits. Participants of this tele-exercise program reported that the convenience and online interaction with a telecoach enhanced their motivation to attend the sessions. This telecoaching (ie, midmanagement) model may become a precursor to self-management and mHealth for optimizing recovery in people with neuromuscular disability.

On the other hand, the expansion of smartphone use and the app design is literally placing sophisticated rehabilitation interventions in the hands of people with disabilities. The potential of mRehab applications include symptom monitoring, real-time data capture, real-time access to information about navigating the community, social connectedness through peer-to-peer support, and bidirectional communication. However, there exist barriers to use of mHealth, such as limited scientific evidence; lack of integration of multiple perspectives and disciplines into workflow; concerns over data confidentiality, privacy, and security; and lack of provisions for reimbursement. Of particular concerns for the disability community is how factors at the human-technology interface can impose barriers to use. Accessibility and usability of mRehab interventions are essential factors that must be considered throughout the app development. An iterative interdisciplinary design process that brings together content, accessibility, and information technology experts with people with disabilities can help ensure the needs and priorities of the disability community.

Many patients after acquiring a disability are unable to receive the optimal amount of rehabilitation and health care services because of a number of challenging barriers. With continuing growth in the Internet and use of smartphones, the development of digital health applications can significantly broaden rehabilitation and health care opportunities for patients. The full potential of digital health technologies to reach a large number of people with disabilities, who exhibit a range of physical and psychosocial secondary health conditions, and provide them with effective dose of interventions has yet to be realized.

\section{Transitions Across the Life Span}

(Moderator: Walter Frontera, MD, PhD, Vanderbilt University; Panelists: Sharon Ramey, PhD, Virginia Polytechnic Institute and State University; Ellen Giarelli, EdD, RN, MS, CRNP, Drexel University; Eric Lenze, MD, Washington University)

The purpose of this session was to examine current evidence and discuss future research needs in the area of rehabilitation across the life span, with a particular emphasis on transitions. Disability has an effect on growth and development, transitions to adulthood, and aging (particularly disabling medical conditions). At the same time, these processes can influence how individuals adapt to the presence of disability and the nature of their health care needs.

The first presentation focused on the need to increase the number of implementation science trials to identify approaches and strategies that work best with a high degree of certainty. Examples of areas in need of this approach include studies on cost-benefit ratio and health disparities. Rapid high-fidelity science is needed to put research into practice more quickly. In a real-world setting, it is important to know if the clinician is familiar with the latest evidence and the best way to effectively deliver care with high efficiency and consistency. We need to understand the barriers and obstacles that prevent research results from being implemented. In other words, why does it take so much time and energy to change practice?

Health care, and specifically rehabilitation for patients with chronic syndromes diagnosed in childhood, including those associated with genetic variants, is best accomplished when it is begun early in life, as soon as a diagnosis is pending, conceptualized as requiring the integration of skills, 
knowledge, and clear intentions of a diverse team, and the team is composed of the patient, health care providers, family members, and other advocates. Transitioning of any kind can be complicated and is always highly personal. Furthermore, lifelong management is complex, requires more health care, and is associated with higher costs. Therefore, we must use models that capture sociocultural, environmental, and health variables and barriers to identify paths to or loci of success. A fundamental goal is promoting the patient's skill at self-surveillance and self-management, including rehabilitation. There are no tricks, magic, or fail-safe; it is hard work that must be individualized and supported.

Aging is associated with significant emotional, cognitive, and/or motivational impairments that interfere with successful rehabilitation interventions. Clinical strategies that focus on patient engagement and therapy intensity can help with behavioral changes that are needed for successful rehabilitation. A model of enhanced medical rehabilitation therapy was presented by Dr Lenze. This model includes a package of motivational and high-intensity therapy steps that physical and occupational therapists can take to maximize both patient engagement and therapy intensity. Effort and progress are reinforced during therapy with direct feedback to the patient, and therapy is linked to goals set by the patients. Older adults receiving therapy from enhanced medical rehabilitation-trained therapists had greater engagement in therapy sessions, higher patient active time, and better functional recovery, compared with patients receiving typical standard-of-care therapy.

\section{Novel Outcomes in Rehabilitation and Integration Into Clinical Care}

(Moderator: Jonathan Bean, MD, Harvard Medical School; Panelists: Brad Dicianno, MD, University of Pittsburgh; Melissa Morrow, PhD, Mayo Clinic; Brian Hafner, PhD, University of Washington)

The purpose of this session was to examine the clinical and scientific relevance of developing novel outcomes in rehabilitation and its potential to favorably affect the changing health care environment. Health care reform and the shifting emphasis on managing health have been coupled with exceptional growth and development in the application of technology and engineering to health measurement. As the mobile health field and technologies evolve, researchers will continuously be presented with challenges in the conceptual design and deployment of clinical trials as well as the conduct of clinical care owing to the vast array of outcomes measures that can be collected.

The Interactive Mobile Health and Rehabilitation (iMHere) system is an example of a mobile health system being used to collect ecological momentary assessment outcomes data among patients with spina bifida (Diciano). Furthermore, wearable sensors monitoring different aspects of health are becoming more widely used in rehabilitation research as a method of capturing real-world outcomes. For example, sensor based outcomes are being used (Morrow) in SCI rehabilitation research, although there are challenges to the integration of "big data" into clinical practice. New approaches to outcomes measurement have also been applied to the development of patient-reported outcomes. National initiatives, such as the Patient Reported Outcomes Measurement Information System, have resulted in rigorous frameworks for developing patient-reported outcomes that can evaluate health outcomes across different patient populations. Efforts using these same methods to develop an item bank specific to measuring prosthetic mobility in people with lower-limb loss were described.

The discussion following the presentations identified a number of issues and challenges. These included a number of general issues, such as (1) the importance of developing a consolidated infrastructure, be that through industry partnerships or academic hubs; (2) using that infrastructure to develop systems that integrate mHealth, wearables, and patient-reported outcomes in efficient ways, so that they complement each other to optimize assessment and monitoring; (3) developing strategies to incorporate these integrated data elements into measurement systems with which patients and clinicians can optimally engage and interact; and (4) the integration of the resulting data into the electronic medical record. Specific needs that were discussed also included (1) developing "standards" or "best practices" for wearable sensor technology akin to what the Patient Reported Outcomes Measurement Information System had done for patient-reported outcomes, (2) developing strategies for extracting the "most important" data from wearable sensors and presenting them in a way that is appropriate for the given stakeholder (patients, practitioners, payers), and (3) using these approaches for more optimal management of self-care and, thus, relieving clinicians of the burden created by interpreting and processing high volumes of data. Finally, integrated leadership in addressing these concerns was viewed as a priority for $\mathrm{NIH}$, especially in cooperation with other relevant agencies such as the Patient-Centered Outcomes Research Institute, the Agency for Healthcare Research and Quality, or the Veterans Health Administration.

\section{Using Data to Drive Discovery}

(Moderator: Ken Ottenbacher, PhD, University of Texas Medical Branch; Panelists: Adrian Hernandez, MD, Duke University; James Graham, $\mathrm{PhD}$, University of Texas Medical Branch; Jennifer Hicks, PhD, Stanford University)

The purpose of this session was to examine the use of data as a means to drive discovery. Using data to drive discovery has been a hallmark of scientific investigation since the 1600s, beginning with the writings of Sir Francis Bacon regarding the modern scientific method. How data have 
been defined and used to generate new knowledge has evolved dramatically since then. The pace has been particularly rapid during the past decade. This revolution is being driven by several factors, including (1) advances in information technology, (2) the development of sophisticated data analytics, and (3) the increased availability and complexity of data. These factors provide opportunities for data integration, exploration, and secondary analysis that did not exist even a few years ago. The NIH "Big Data" program, referred to as BD2K (Big Data to Knowledge) and launched in 2012, is a reflection of the data revolution and its impact on biomedical and health care sciences. For the fields of rehabilitation medicine and disability sciences to fully participate in the research opportunities associated with using data to drive discovery, there is a need to raise awareness and build research capacity.

Significant opportunities exist for data exploration and analyses in existing administrative and federal data sets, including resources supported by the NIH specifically designed for rehabilitation investigators-for example, Center for Large Data Research \& Data Sharing in Rehabilitation. In addition, the Mobilize Center, a NIH BD2K Center of Excellence, is using modern data science tools to integrate and analyze information from wearable sensors, research laboratories, and clinics to understand and improve human mobility, for example, to improve treatment for patients with CP. The NIH-funded National Center for Simulation in Rehabilitation Research provides the worldwide rehabilitation research community with a common platform for sharing data and models that describe movement. Additional opportunities for discovery exist using large administrative or public use databases such as Medicare claims and assessment files and US Census data (including data related to the Affordable Care Act and health care reform). There are rapidly emerging opportunities for information sharing and secondary analyses of data from completed studies associated with recent federal data sharing and archiving mandates. The use of electronic health records and the creation of large data networks and a health system collaboratory represent yet another opportunity to use clinical data with an emphasis on patient-reported and patient-centered outcomes. Examples included the NIH Collaboratory, the National Heart Lung and Blood Institute's Heart Failure Research Network, and the PCORnet: National Patient-Centered Clinical Research Network, which includes data from more than 100 million people.

Using data to drive discovery is an important and rapidly expanding area of research with enormous potential to advance rehabilitation science and patient care. This session provided an introduction to the emerging discipline of data science and its application and implications for rehabilitation research. A better understanding of data science will help rehabilitation clinicians, administrators, and investigators accomplish the Conference's goal of "Moving the Field Forward."

\section{Preventing Secondary Disability}

(Moderator: Diane Damiano, PhD, PT, NIH Clinical Center; Panelists: Greg Hicks, PhD, University of Delaware; Diann Gaalema, PhD, University of Vermont; Sara Mulroy, PhD, Rancho Los Amigos National Rehabilitation Center)

This session focused on major issues in prevention of secondary disability across 4 distinct populations: children with $\mathrm{CP}$, elderly adults with low back pain, adults with SCIs, and adults recommended for cardiac rehabilitation programs. Even with this diversity, many similarities were seen across the presentations.

The scientific basis across populations for addressing secondary impairments focused on the identification of modifiable factors that if addressed would improve outcomes in terms of health and functioning for these individuals. For children with CP, the focus was on physical activity throughout the life span to preserve and maintain optimal muscle and brain functioning. A particular emphasis was the need to intervene very early in life to limit the development of secondary changes caused by the inactivity imposed by the brain lesion. For elderly adults with low back pain, trunk muscle integrity has been identified as a key modifiable factor in this population that can reduce pain. Interestingly, pain was previously thought to be an almost inevitable part of normal aging, so much so that older adults were typically excluded from studies on low back pain. The patients at highest risk for poor outcomes after cardiac surgery are often the ones who are least likely to attend rehabilitation programs that have been shown to be efficacious in improving these outcomes. It is important to identify why these individuals chose not to attend, with the goal of devising strategies to improve their participation. Compliance with rehabilitation or with long-term behavioral health changes was a theme that resonated across speakers and the audience. Efforts to incentivize patients to participate, while expensive, may reduce health care costs tremendously if successful. Another patient population with secondary disability is that of individuals with SCIs with shoulder injuries. Using sophisticated biomechanical analyses, movement patterns that markedly diminished shoulder pain have been identified, again showing that research is needed on modifiable factors that enable people to remain or increase their ability to be mobile, whether it is in a wheelchair or walking in the community. It was emphasized that patients should have greater involvement in our research, so we can learn their concerns and challenges and their individual factors that make them more likely to have adverse health outcomes. In some instances, it can be socioeconomic status; in $\mathrm{SCI}$ in southern California, living in a violent neighborhood 
increased the chances markedly of having a SCI, both of which present very unique and specific public health challenges in addition to the scientific challenges.

Future recommendations for research are to better engage our patients and their needs into our research efforts and to be more open to alternative methodologies besides RCTs to find cost-effective methods to help people maintain their health across the life span. From a more translational science perspective, we need to know more about mechanisms leading to pain across disorders and continue to explore biomechanical and motor learning/training strategies to improve functionality and reduce pain rather than masking the chronic pain with medication. For children with $\mathrm{CP}$, more effective early intervention strategies need more investigation while, at the same time, the intersection of aging with a disability is also a major gap in the literature. Finally, secondary disability is hardly secondary in cost, duration, and importance to patients. However, because it occurs as a result of a primary injury, these could theoretically all be avoidable or at least modifiable, and this is where rehabilitation research is needed.

\section{Development of an NIH Rehabilitation Research Plan}

(Presenters: Dr Alison Cernich and Dr Lyn Jakeman)

The session covered the development of the new NIH Rehabilitation Research Plan. The intent of the plan is to detail research priorities that are of interest to a large group of the Institutes and Centers in the NIH that invest in rehabilitation research. A trans-NIH Medical Rehabilitation Research Coordinating Committee began development of the plan in 2015. They developed the priorities in the plan in consultation with the National Advisory Board on Medical Rehabilitation Research and the directors of the NIH Institutes and Centers. NIH published a draft of the plan asking for public comment in November of 2015 and revised the plan based on that input.

Through feedback received through the request for public input, the Medical Rehabilitation Research Coordinating Committee modified the plan to include the development of new methods to foster interdisciplinary research, placing greater emphasis on health disparities, and broadening the avenues for development of new technologies. As a result of the comments, the Medical Rehabilitation Research Coordinating Committee added 2 priority areas and revised and refined other priority areas. The final plan includes 6 priority areas: Rehabilitation Across the Life Span, Family and Community, Technology Use and Development, Translational Science, Research Design and Methodology, and Research Capacity and Infrastructure. The plan was intended to be final in June, and a town hall meeting at the conference provided the final opportunity for feedback to the Medical Rehabilitation Research Coordinating Committee before the plan was published.

\section{Authors' Note}

Reprinted with permission from: Frontera WR, Bean JF, Damiano D, Ehrlich-Jones L, Fried- Oken M, Jette A, Jung R, Lieber RL, Malec JF, Mueller MJ, Ottenbacher KJ, Tansey KE, Thompson A. Rehabilitation research at the National Institutes of Health: Moving the field forward (executive summary). American Journal of Physical Medicine and Rehabilitation, Volume 96, Issue 4, pages 211-220. April 2017. http://journals.lww.com/ajpmr.

\section{Acknowledgments}

Six institutes at the National Institutes of Health (NIH) cosponsored this conference, including the Eunice Kennedy Shriver National Institute of Child Health and Human Development, the National Institute of Nursing Research, the National Institute of Biomedical Imaging and Bioengineering, the National Center for Complementary and Integrative Health, the National Institute on Deafness and other Communication Disorders, and the Office of Disease Prevention.

\section{Declaration of Conflicting Interests}

The authors declared no potential conflicts of interest with respect to the research, authorship, and/or publication of this article.

\section{Funding}

The authors disclosed receipt of the following financial support for the research, authorship, and/or publication of this article: Financial disclosure statements have been obtained, and no conflicts of interest have been reported by the authors or by any individuals in control of the content of this article. 